\section{Genetic Markers in a Medieval Case of Ankylosing Spondylitis}

\section{To the Editor:}

Ankylosing spondylitis (AS) is a chronic rheumatic autoimmune disease that affects mainly the sacroiliac joints and spine, where it causes ankylosis through processes of inflammation and ossification. Although the exact accurate etiology of AS is unknown, the disease's development has been shown to be influenced by genetic factors. The HLA-B27 allele is the strongest genetic marker associated with $\mathrm{AS}^{1}$, but there are other genes both within and outside the MHC that are involved in the development of AS. The most significant laboratory test in the diagnosis of AS involves detecting the presence of the allele HLA-B27, because $90-95 \%$ of patients with AS have this allele ${ }^{2}$. Nevertheless, this allele is found in about $10 \%$ of the world's population; of them, only $5 \%$ ultimately develop this disease ${ }^{2}$. This means that when there are several radiological and clinical symptoms, detecting the allele HLA-B27 is not enough to diagnose the disorder.

The aim of our study was to identify several genetic markers associated
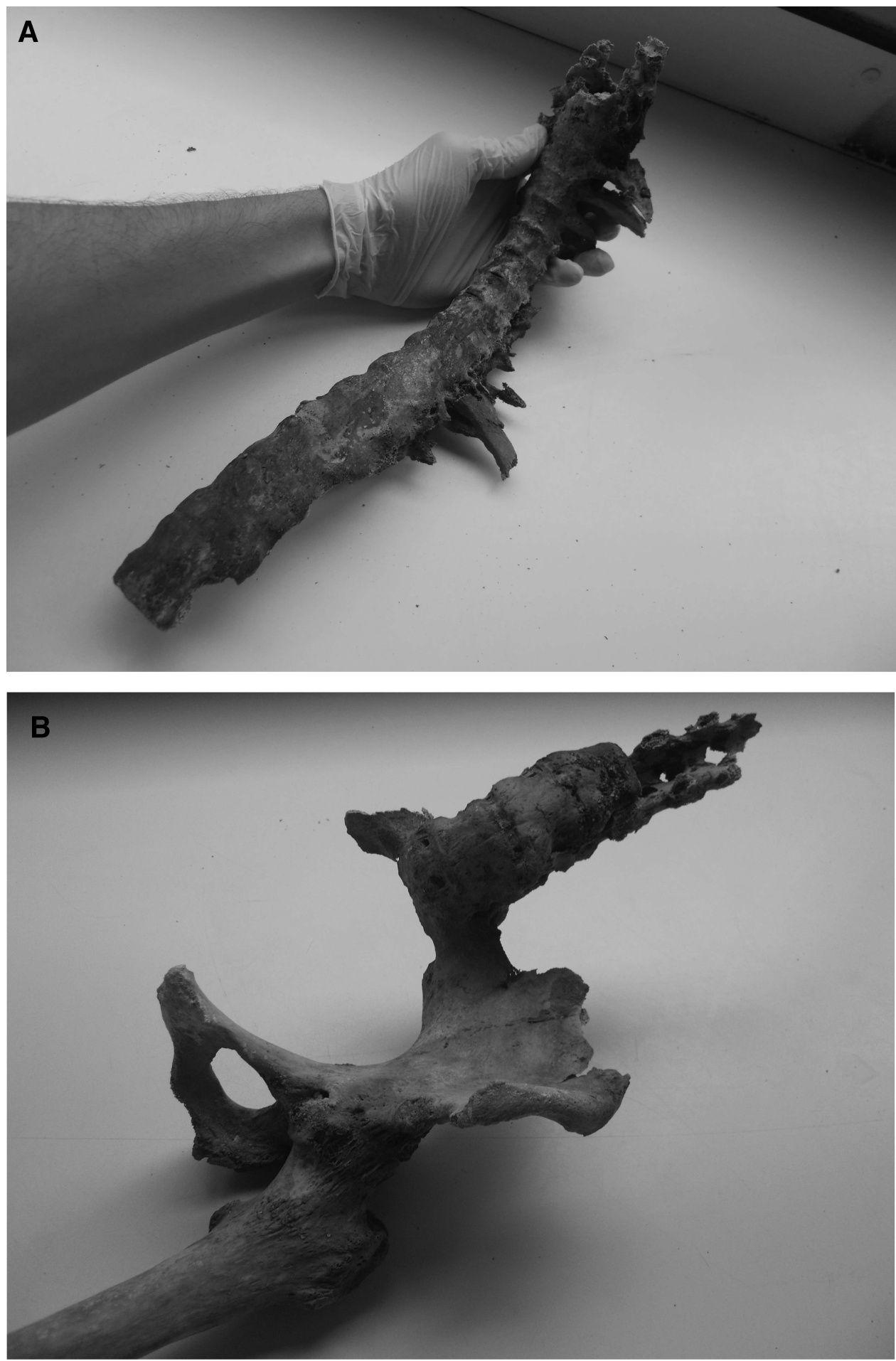

Figure 1. Human bone remains belonging to a medieval individual (16th century) from Santa María in Vitoria-Gasteiz (Basque Country, Spain). The diagnostic bone manifestations of AS are shown: A. Fused bamboo-shaped spinal column. B. Bilateral fusion of the sacroiliac joints and of the lumbosacral region 
with the development of AS [HLA-B27 allele and single-nucleotide polymorphisms (SNP) in interleukin $23 \mathrm{R}(I L-23 R)$ and ERAP1 genes] in a medieval burial site (16th century Basque Country, Spain) that shows human remains with morphological and radiological manifestations of AS, i.e., a bamboo-shaped spinal column and bilateral fusion of the sacroiliac joints and of the lumbosacral region (Figure 1). It was identified as a female (based on the morphology and the presence of the amelogenine gene). This is the first ancient DNA (aDNA) study, to our knowledge, to address the nuclear markers associated with the development of AS, instead of focusing only on the presence/absence of the HLA-B27 allele ${ }^{3,4}$. The identification of nuclear mutations in an ancient individual with unequivocal signs of having had AS can help to define a set of mutations that contribute to the early diagnosis of this disease.

We extracted DNA from 3 ribs to obtain the sequence of exons 2 and 3 of $H L A-B$ gene and determine the SNP in $I L-23 R$ and ERAPI genes by using 10 pairs of primers that we designed (Table 1). The HLA-B27 gene records a high degree of genetic polymorphism, mainly in exons 2 and 3 of the gene's 8 exons ${ }^{5}$. There are 105 known subtypes encoded by 132 alleles, defined on the basis of the differences in the nucleotide sequence ${ }^{5}$. The present study involved the extraction and analysis of aDNA, while taking the usual precautions in aDNA studies to avoid any possible contamination as negative controls for extraction and polymerase chain reaction amplification. Further authentification criteria are cloning of amplified products (at least 10 clones for each amplified product), analysis in triplicate, and genetic typing of archaeologists who handled these bones, to identify any possible contamination in the sample ${ }^{6}$. On the other hand, the mitochondrial DNA of this individual (revised Cambridge Reference Sequence) was not found in any of the researchers involved in the present study, therefore contamination was avoided $^{6}$

We determined that this individual was homozygous for HLA-B27 allele. This implies a risk of developing AS 3-fold higher than in heterozygous counterparts ${ }^{7}$. The consensus sequence obtained has shown that the individual has the $H L A-B^{*} 27: 90: 01$ subtype, for which there are no prior studies; but we may propose a possible link to AS.
The analysis of the SNP within the $I L-23 R$ gene associated with AS has determined that this individual presented the derived variant (C) rs2201841 and the ancestral variants (G) rs11209026 and rs11209032, which are associated with AS. Further, cloning has been used to confirm that the individual is a homozygote for the 3 SNP. Although the biological effect of these variants in the expression and function of $I L-23 R$ is currently unknown, it seems clear that the SNP within this gene are major factors in the development of $\mathrm{AS}^{8,9}$.

The SNP studied within the ERAP1 gene in this medieval individual has the derived variant (C) rs27044 and the ancestral variants (T) rs30187 and rs2287987. All 3 SNP are homozygous. In the case of the individual studied, who is positive for the HLA-B27 allele, the SNP analyzed could lead to the malfunction of the ERAP1 gene, which would affect the presentation of antigens by the class I molecules of the MHC such as HLA-B27, thus entailing a greater risk of developing $\mathrm{AS}^{8}$.

Although there are diverse studies associating different genes with AS, an analysis of several nuclear genetic markers (HLA-B27 gene and SNP in the $I L-23 R$ and ERAP1 genes) in an individual with unmistakable signs of AS has allowed us to confirm the implication of $I L-23 R$ and $E R A P 1$ genes in the development of AS. We also propose that the subtype $H L A-B * 27: 90: 01$ as a possible genetic marker associated with the disease. Given the lack of clear differential diagnostic criteria for early stages of AS, the determination of a haplotype associated with the disease will contribute to the early diagnosis of AS and other related disorders within the spondyloarthropathies.

IMANOL MARTÍN LAZA, MS; MONTSERRAT HERVELLA, PhD; CONCEPCIÓN DE-LA-RÚA, PhD, Department of Genetics, Physical Anthropology, and Animal Physiology, Faculty of Science and Technology, University of the Basque Country (UPV-EHU), Biscay, Spain. Address correspondence to I. Martín Laza, Department of Genetics, Physical Anthropology, and Animal Physiology, UPV/EHU, Barrio Sarriena s/n 48940 Leioa, Bizkaia, Spain.

E-mail: imanol.martinl@ehu.es

Table 1. Sequence of the primers used for amplifying exons 2 and 3 of the HLA-B27 gene and SNP in $I L-23 R$ and ERAP1 genes, together with the annealing temperature (T) corresponding to each pair of primers and the size of the amplification product obtained.

\begin{tabular}{|c|c|c|c|}
\hline Primer & Primer Sequence (5' to $\left.3^{\prime}\right)$ & $\mathrm{T}\left({ }^{\circ} \mathrm{C}\right)$ & $\begin{array}{c}\text { Length of } \\
\text { Amplification (pb) }\end{array}$ \\
\hline HLAB2-1F* & GCC GCG AGT CCG AGA GA (17) & 65 & 117 \\
\hline HLAB2-1R* & GGC CTC GCT CTG GTT GTA (18) & & \\
\hline HLAB2-2F & CCG GAG TAT TGG GAC CG (17) & 65 & 67 \\
\hline HLAB2-2R & GGC CTC GCT CTG GTT GTA G (19) & & \\
\hline HLAB3-2F & GGG CAG GGT CTC ACA CCC TCC (21) & 65 & 61 \\
\hline HLAB3-2R & GAT GTA ATC CTT GCC GTC GTA (21) & & \\
\hline HLAB3-3F & GGA TTA CAT CGC CCT GAA CG (20) & 60 & 92 \\
\hline HLAB3-3R & TCC ACG CAC TCG CCC TCC AGG T (22) & & \\
\hline IL23R-rs2201841-F & GTG ATG ATT TGT GAC AGT AGT A (22) & 60 & 69 \\
\hline IL23R-rs2201841-R & AAG TGC TGG GCT TAC AGG CA (20) & & \\
\hline IL23R-rs11209026-F & CTT TGA TTG GGA TAT TTA AC (20) & 55 & 86 \\
\hline IL23R-rs11209026-R & CAT ATA CAT GTA GTC TAA ATC AG (23) & & \\
\hline IL23R-rs11209032-F & GCT ATC CTG ACA ATT CCT C (19) & 53 & 83 \\
\hline IL23R-rs11209032-R & CTT CAA GCT GAA TTG CA (17) & & \\
\hline ERAP1-rs2287987-F & ATG AGC TTA TAC CTG GTG AGC (21) & 60 & 32 \\
\hline ERAP1-rs2287987-R & AGT CTT CTG CAT CAA GTA AGC (21) & & \\
\hline ERAP1-rs30187-F & GCT CTT GCT TCA TGT GTA CA (20) & 62 & 87 \\
\hline ERAP1-rs30187-R & CCA TGA TGA ACA CTT GGA CAC (21) & & \\
\hline ERAP1-rs27044-F & AGC CTT CTG CCC TCT GTA (18) & 58 & 110 \\
\hline ERAP1-rs27044-R & GCA GAC ATG GAC AGA CGA G (19) & & \\
\hline
\end{tabular}

* Primer from Dominguez, et al, Immunogenetics, 1992. SNP: single-nucleotide polymorphism; IL: interleukin. 


\section{REFERENCES}

1. Maksymowych WP. Update in spondyloarthropathy. Arthritis Rheum 2004;51:143-6.

2. Brown MA. Breakthroughs in genetic studies of ankylosing spondylitis. Rheumatology 2008;47:132-7.

3. Haak W, Gruber P, Rühli FJ, Böni T, Ulrich-Bochsler S, Frauendorf E, et al. Molecular evidence of HLA-B27 in a historical case of ankylosing spondylitis. Arthritis Rheum 2005;52:3318-9.

4. Leden I, Götherström A, Drenzel L, Svensson B. HLA-B27 sequences identified in a mediaeval skeleton with ankylosing spondylitis. Ann Rheum Dis 2009;68:757-8.

5. Khan MA. Remarkable polymorphism of HLA-B27: An ongoing saga. Curr Rheumatol Report 2010;12:337-41.

6. Gilbert MT, Willerslev E. Authenticity in ancient DNA studies. Med Secoli 2006;18:701-23.

7. Jaakkola E, Herzberg I, Laiho K, Barnardo MC, Pointon JJ, Kauppi $\mathrm{M}$, et al. Finnish HLA studies confirm the increased risk conferred by HLA-B27 homozygosity in ankylosing spondylitis. Ann Rheum Dis 2006;65:775-80.

8. Burton PR, Clayton DG, Cardon LR, Craddock N, Deloukas P, Duncanson A, et al. Association scan of 14,500 nonsynonymous SNPs in four diseases identifies autoimmunity variants. Nat Genet 2007;39:1329-37

9. Sáfrány E, Pazár B, Csögeni V, Járomi L, Polgár N, Sipeky C, et al. Variants of the IL23R gene are associated with ankylosing spondylitis but not with Sjögren syndrome in Hungarian population samples. Scan J of Immunol 2009;70:69-74.

10. Dominguez O, Coto E, Martinez-Naves E, Choo SY, López-Larrea C. Molecular typing of HLA-B27 alleles. Immunogenetics 1992;36:277-82.

J Rheumatol 2016;43:3; doi:10.3899/jrheum.151170 